\title{
ResearchOnline@JCU
}

This is the Accepted Version of a paper published in the journal Lancet:

Wang, Yutang (2014) Renal denervation for resistant hypertension: the Symplicity HTN-1 study. Lancet, 383 (9932). $1885-1885$.

http://dx.doi.org/10.1016/S0140-6736(14)60912-0 


\section{Renal denervation for resistant hypertension-the Symplicity HTN-1 study}

Yutang Wang, PhD

The Vascular Biology Unit, Queensland Research Centre for Peripheral Vascular Disease, School of Medicine and Dentistry, James Cook University, Townsville, Queensland 4811, Australia

Corresponding author: Yutang Wang. The Vascular Biology Unit, Queensland Research Centre for Peripheral Vascular Disease, School of Medicine and Dentistry, James Cook University, Townsville, Queensland 4811, Australia. Tel: +61 74781 3155; fax: +61 74781 3652; E-mail address: yutangwang000@gmail.com. 
To the editor

The effect of RDN on renal artery stenosis was not well investigated in Henry Krum and colleagues' study ${ }^{1}$. Only 18 patients underwent angiography at 14-30 days, and 14 underwent magnetic resonance angiography at 6 months. Therefore, the follow-up rate was very low in this cohort of 153 patients. It was not reported that how many patients underwent renal artery imaging at 12, 24, and 36 months. Nor was reported the exact imaging method used at these time points. Ultrasonography has limitations in detecting renal artery stenosis., ${ }^{2,3}$

It is of concern that estimated glomerular filtration rate (eGFR) decreased $(p=0.05)$ and the creatinine concentrations in the serum progressively increased $(p=0.05) .{ }^{1}$ In addition, the effect of RDN on renal function was not clearly described. 28 patients had a decrease in eGFR of more than $25 \%$ after $\mathrm{RDN}^{1}$. However, it was not reported how many patients were followed up at each time point; nor was reported to what extent the eGFR was decreased in each patient. It would be more informative if the authors could provide the eGFR values for these 28 patients over this 3-year follow-up in a table and also how many patients showed a clinically significant increase in creatinine concentrations at each time point. The authors can not conclude that RDN is safe.

\section{CONFLICT OF INTEREST}

The authors declare that they have no conflict of interest. 


\section{REFERENCES}

1 Krum H, Schlaich MP, Bohm M, et al. Percutaneous renal denervation in patients with treatment-resistant hypertension: final 3-year report of the Symplicity HTN-1 study. Lancet 2013; Doi: 10.1016/S0140-6736(13)62192-3.

2 Zhang HL, Sos TA, Winchester PA, Gao J, Prince MR. Renal artery stenosis: imaging options, pitfalls, and concerns. Prog Cardiovasc Dis 2009; 52: 209-19.

3 Lao D, Parasher PS, Cho KC, Yeghiazarians Y. Atherosclerotic renal artery stenosis-diagnosis and treatment. Mayo Clin Proc 2011; 86: 649-57. 